\title{
Objective evaluation of acupuncture treatment in patients with cervical spondylosis by pulse diagnosis device
}

\author{
Haifang Zhang, Zhifeng $\mathrm{Yu}^{\mathrm{a}}$, Linjie Fu and Xiaozuo Lu \\ School of Chinese Medicine and Engineering, Tianjin University of Traditional Chinese Medicine, Tianjin, 300193, \\ China
}

\begin{abstract}
To study changes in pulse diagram parameters (PDP) in patients with cervical spondylosis (CS) before and after acupuncture treatment, explore the characteristics of PDP and the relationship between PDP changes and therapeutic effectiveness, and provide evidence for outcome prediction and objective evaluation of CS treatment before and after acupuncture treatment.Patients with CS were treated with acupuncture and measured with a pulse acquisition device based on image (PADBI) before the first and after the tenth acupuncture sessions. PDP changes from before until after the acupuncture sessions and patient impressions were analyzed to judge the effect of acupuncture treatment. The PDP values in effective patients were closer to normal values. This indicated that Qi stagnation and blood stasis of the patients were improved. The PDP changes from before to after the first acupuncture treatment were more obvious than those from before to after the tenth acupuncture treatment. This result indicates that the speed of symptom improvement decreased significantly after several acupuncture courses. Analysis of correlation between efficacy and PDP showed that the changes in PDP in five patients was abnormal, which mainly manifested as values of $h 1, u, p$, $\mathrm{Pp}$, and $\mathrm{t} 1$, and no significant changes or differences were increased with standard values. This indicated that the symptoms of CS were not improved in these patients.PADBI can provide evidence for outcome prediction of acupuncture treatment in patients with CS. PADBI can provide evidence for objective evaluation of acupuncture treatment of CS.
\end{abstract}

\section{Introduction}

Pulse feeling is one of the important diagnostic methods in Traditional Chinese Medicine (TCM) for obtaining physiological and pathological information. It is difficult to identify pulse patterns accurately because pulse conditions contain a lot of information. The traditional method of pulse feeling with the fingers is subjective and the results can vary from person to person.

Cervical spondylosis (CS) is caused by degeneration and prolapse of the cervical intervertebral discs and cervical joints, which directly compress cervical spinal nerves, vertebral arteries and the spinal cord. The major clinical manifestations are pain along the neck and shoulder, upper extremity numbness, and radiating pain[1-3]. CS is similar to that of Qi stagnation and blood stasis in terms of TCM theory. The etiology of CS is multifactorial and involves poor posture, anxiety, depression, neck strain, and sports or occupational activities[4]. Conventional medical treatments for CS neck are

\footnotetext{
${ }^{\mathrm{a}}$ Corresponding author: fengzhiu86@hotmail.com
} 
limited by their modest effectiveness. TCM interventions have been widely used for the management of CS neck pain[5-8] and acupuncture is one of the most popular managements. Many clinical studies have been conducted to evaluate the efficacy of acupuncture[9-11]. While, modern medicine has many diagnostic methods for CS, TCM diagnoses mainly rely on auscultation-olfaction, interrogation, and palpation, especially the pulse feeling. Because of differences in understanding and clinical experience in traditional pulse feeling, the objectification of pulse information is more important.

To study the significance of pulse acquisition device based on image (PADBI) in the objective evaluation of acupuncture treatment for CS, we used TD-III-Type PADBI to measure PDP changes before and after acupuncture treatment in 50 patients with CS.

\section{Materials and methods}

\subsection{Study samples}

This study included 50 patients suffering from CS being treated with acupuncture at the Chinese Medicine outpatient clinic, Affiliated Baokang Hospital of Tianjin University of TCM between October 2013 and March 2014. The group was composed of 38 women and 12 men, ranging from 18 to 78 years old, with an average age of 44.08 . Among the 50 patients, 22 patients were under 40,19 patients were 41-60 years old, and 9 patients were aged 61 years or older. All patients understood and agreed with the study items. The study design was approved by Medical Ethics Committee of Tianjin University of Traditional Chinese Medicine.

\subsection{Inclusion criteria}

Patients were included if they were men or women aged between 18 and 80, fit the diagnostic criterion established in the 2nd Symposium Meeting on CS[12] and agreed to sign the informed consent and complete treatments on time.

\subsection{Exclusion criteria}

Patients with allergic constitution and needle syncope were excluded. Patients were excluded if they had a serious primary disease such as severe hypertension, cardiovascular and kidney diseases, hematopoietic system diseases, and other life-threatening diseases, or if they were suffering from mental disorders. Patients unwilling to participate in the study or were participating other medical trials were excluded.

\subsection{Diagnostic criteria for CS}

According to the 2nd Symposium Meeting on Cervical Spondylosis, the general diagnostic principle for CS was diagnosed by clinical manifestations and X-rays. If patients presented with typical CS clinical manifestations, but X-ray did not reveal abnormalities and other diseases were excluded, then patients could still be diagnosed as having CS. Patients without clinical chief complaints and signs, but with abnormal X-rays should not be diagnosed with CS.

\subsection{Treatment}

Acupoints selected were: Jiaji (EX-B2), Dazhui (BU 14), Fengchi (GB 20), Xinshe (EX-HN 21), Baihui (BU 20), Sishencong (EX-HN 1), Quchi (LI 11), Shousanli (LI 10), and Hegu (LI 4). Sterile single-use acupuncture needles $(40 \mathrm{~mm}$ in length and $0.30 \mathrm{~mm}$ in diameter; Hwato brand, Suzhou Medical Products Factory Co., Suzhou, China) were inserted into Jiaji (EX-B2) points to a depth of 20 to $30 \mathrm{~mm}$ at an angle of 75 degrees. The direction of needle insertion in Fengchi (GB 20) was 
orientated towards the contralateral canthus (to a depth of 25 to $30 \mathrm{~mm}$ ). Points in the head were inserted into the skin to the subcutaneous tissue at an angle of 15 degrees to the point surface. Other points were perpendicularly inserted. The inserted needles were manually manipulated until the patient felt numbness or the acupuncture sensation known as "De Qi." Needles were retained in the points for $30 \mathrm{~min}$. Sessions were administered once every other day with 10 sessions constituting one course.

\subsection{Trial design}

Fifty subjects with CS neck pain from outpatient clinics were recruited and treated with acupuncture. Then, their PDP was measured with PADBI before and after the first and the tenth acupuncture treatments. The assessment scale for cervical spondylosis used in the study was based on the score diagram of treatment on diseases of lumbar vertebrae[13] by the Japanese Orthopaedic Association in 1984 which can objectively reflect improvements in cervical function, and quantitatively evaluate the efficacy of patients with CS.

One course of treatment with acupuncture was given to each patient. The doctors performing all therapies had extensive traditional Chinese clinical experience. PDP was recorded before and after the first acupuncture treatment and before and after the tenth acupuncture treatment. The parameters recorded and analysis were extracted independently by other researchers.

Essential information including age, case history, and treatment history were collected. All of the findings in the history and physical examination were recorded in a standard TCM assessment form. Patients were asked to remain relaxed and were pulse-diagnosed by a TCM doctor. Their pulse type of the left hand was recorded before acupuncture treatment. PADBI was placed on the left radial artery and the detection software was started. Pulse signals were recorded for $30 \mathrm{~s}$ before acupuncture treatment. Then, the acupuncture treatment was carried out for $30 \mathrm{~min}$. Patients rested for 1-2 min after withdrawing the needle, and pulse signals were recorded for another $30 \mathrm{~s}$.

\subsection{Statistical analyses}

SPSS 18.0 statistical software (IBM Corp., Armonk, NY, USA) was used for statistical analyses. Pulse measurements are expressed as mean \pm standard deviation. Comparisons for pulse were analyzed by time domain analyses. The inner-group comparison was analyzed by t-test. A P value $<$ 0.05 was considered statistically significant.

\section{Results}

We used TD-III-Type PADBI to measure changes in the PDP (p, u, t1, h1, Pp) before and after acupuncture treatment in 50 patients with CS. The $\mathrm{P}$ angle is the angle of the main wave, and can reflect changes in vascular elasticity and condition of blood flow. Decreased $\mathrm{P}$ angle indicates ameliorated vascular elasticity, normal circulation of Qi and blood, unobstructed blood flow, and little myocardial ischemia. The $\mathrm{U}$ angle is the angle of ascent, and can reflect changes in vascular elasticity and blood viscosity. Increased $U$ angle indicates better vascular elasticity and healthier Qi and blood circulation. $\mathrm{t} 1$ corresponds to the rapid ejection period of left ventricle. Decreases in $\mathrm{t} 1$ after treatment indicate that Qi and blood are sufficient and indicate normal function. h1 is the main wave height, and can reflect the ejection function of the left ventricle and arterial compliance. Increases in h1 indicate that the left ventricular ejection function is enhanced, arterial compliance is improved, Qi and blood are sufficient, and Qi and blood circulation are healthy. The Pp value is the numerical difference between two peaks in the pulse wave. The numerical difference in normal human is commonly less than $0.12 \mathrm{~s}$. Decreases in Pp after treatment indicate stable pulse wave rhythm and stable circulation of Qi and blood.

The pulse biology before and after the first treatment, before and after the tenth treatment, and before the first treatment and after the tenth treatment were analyzed (Tables 1-3). The PDP values of 
effective patients were closer to normal. This indicated that the Qi stagnation and blood stasis of these patients were improved. The data showed that the changes in PDP values from before to after the first acupuncture treatment were more obvious, than those from before to after the tenth acupuncture treatment. Furthermore, patients entered a consolidating adjustment period after one course of treatment, which was also effective for acupuncture treatment, although the speed of symptom improvement decreased significantly. Therefore, PADBI has important significance on the objective evaluation of acupuncture treatment in CS.

We found that the PDP ( $h 1, \mathrm{u}, \mathrm{p}, \mathrm{Pp}, \mathrm{t} 1$ ) values were closer to healthy after the tenth treatment in patients whose PDP tended to be healthy after the first treatment. Although the changes in PDP from before to after the tenth acupuncture treatment were less pronounced, the values after the tenth treatment were closer to normal than the values after the first treatment. This is because of increasing differences value in the assessment scale.

The analysis of correlation between efficacy and PDP showed changes of PDP in five patients that indicated ineffective treatment. This manifested as the values of $h 1, \mathrm{u}, \mathrm{p}, \mathrm{Pp}, \mathrm{t} 1$ remaining constant or increasing (Table 4). This indicated that the circulation of Qi and blood were not adjusted. The symptoms of Qi stagnation and blood stasis and arthralgia were not improved.

Table 1. Comparison of pulse diagram parameters before and after the first session acupuncture treatment $(\mathrm{x} \pm \mathrm{s})$.

\begin{tabular}{cccccc}
\hline Index & Before treatment & After treatment & t value & P value & Standard value \\
\hline Pp (s) & $0.22 \pm 0.24$ & $0.12 \pm 0.16 \mathrm{a}$ & 2.748 & 0.008 & $<0.12$ \\
t1 (s) & $0.13 \pm 0.06$ & $0.10 \pm 0.03 \mathrm{a}$ & 3.019 & 0.004 & $0.07-0.11$ \\
$\mathrm{~h} 1(\mathrm{~mm})$ & $13.52 \pm 9.10$ & $17.10 \pm 7.78 \mathrm{~b}$ & -2.429 & 0.019 & $9-22$ \\
u (deg.) & $65.11 \pm 2.55$ & $86.51 \pm 1.15 \mathrm{a}$ & 3.693 & 0.001 & $80-87$ \\
p (deg.) & $69.82 \pm 43.23$ & $31.87 \pm 21.73 \mathrm{a}$ & 5.734 & 0.000 & $19-42$ \\
\hline
\end{tabular}

Notes: Pp value is the numerical difference between adjacent peaks of the pulse wave. The numerical difference of normal human is commonly less than $0.12 \mathrm{~s}$. $\mathrm{t} 1$ corresponds to the rapid ejection period of the left ventricle. $\mathrm{h} 1$ is the main wave height, it can reflect the eject function of left ventricle and arterial compliance. $U$ angle is the angle of ascent, and can reflect changes in vascular elasticity and viscosity of blood. P angle is the angle of the main wave, and can reflect changes in vascular elasticity and condition of blood flow. a $\mathrm{P}<0.01$, vs before the first session treatment, $\mathrm{b} \mathrm{P}<0.05$, vs before the first session treatment.

Table 2. Comparison of pulse diagram parameters before and after the tenth session acupuncture treatment $(\mathrm{x} \pm \mathrm{s})$.

\begin{tabular}{cccccc}
\hline Index & Before treatment & After treatment & t value & P value & Standard value \\
\hline h1 $(\mathrm{mm})$ & $17 \pm 7$ & $21 \pm 10 \mathrm{a}$ & -2.651 & 0.011 & $9-22$ \\
$\mathrm{p}($ deg.) & $34 \pm 26$ & $29 \pm 21 \mathrm{a}$ & 1.409 & 0.165 & $80-87$ \\
\hline
\end{tabular}

Notes: h1 is the main wave height, it can reflect the eject function of left ventricle and arterial compliance. P angle is the angle of the main wave, and can reflect changes in vascular elasticity and condition of blood flow. a $\mathrm{P}$ $<0.05$, vs before the tenth session treatment.

Table 3. comparison of pulse diagram parameters before the first session and after the tenth session acupuncture treatment $(\mathrm{x} \pm \mathrm{s})$.

\begin{tabular}{cccccc}
\hline Index & Before treatment & After treatment & t value & P value & Standard value \\
\hline Pp $(\mathrm{s})$ & $0.22 \pm 0.24$ & $0.08 \pm 0.10 \mathrm{a}$ & 3.743 & 0.000 & $<0.12$ \\
$\mathrm{t} 1(\mathrm{~s})$ & $0.13 \pm 0.06$ & $0.11 \pm 0.03 \mathrm{a}$ & 2.849 & 0.006 & $0.07-0.11$ \\
$\mathrm{~h} 1(\mathrm{~mm})$ & $13.52 \pm 9.10$ & $20.56 \pm 9.67 \mathrm{a}$ & -3.906 & 0.000 & $9-22$ \\
u (deg.) & $65.11 \pm 2.55$ & $86.53 \pm 0.96 \mathrm{a}$ & 4.016 & 0.000 & $80-87$ \\
$\mathrm{p}$ (deg.) & $69.82 \pm 43.23$ & $28.68 \pm 21.07 \mathrm{a}$ & 6.143 & 0.000 & $19-42$ \\
\hline
\end{tabular}

Notes: Pp value is the numerical difference between adjacent peaks of the pulse wave. The numerical difference of normal human is commonly less than $0.12 \mathrm{~s}$. $\mathrm{t} 1$ corresponds to the rapid ejection period of the left ventricle. $\mathrm{h} 1$ is the main wave height, it can reflect the eject function of left ventricle and arterial compliance. $U$ angle is the angle of ascent, and can reflect changes in vascular elasticity and viscosity of blood. $\mathrm{P}$ angle is the angle of the main wave, and can reflect changes in vascular elasticity and condition of blood flow. a $\mathrm{P}<0.01$, vs before the first treatment. 
Table 4. analysis of correlation between efficacy and pulse diagram parameters $(\mathrm{x} \pm \mathrm{s})$.

\begin{tabular}{cccccc}
\hline Item & $\mathrm{Pp}(\mathrm{s})$ & $\mathrm{t} 1(\mathrm{~s})$ & $\mathrm{h} 1(\mathrm{~mm})$ & $\mathrm{u}($ deg. & $\mathrm{p}($ deg. $)$ \\
\hline Marked effect & $0.06 \pm 0.10$ & $0.11 \pm 0.01$ & $22.49 \pm 3.51$ & $86.72 \pm 0.68$ & $22.96 \pm 5.83$ \\
Effect & $0.07 \pm 0.06$ & $0.11 \pm 0.03$ & $19.84 \pm 5.16$ & $86.46 \pm 1.36$ & $32.62 \pm 3.38$ \\
No effect & $0.23 \pm 0.24$ & $0.13 \pm 0.06$ & $13.53 \pm 9.20$ & $65.09 \pm 2.58$ & $70.61 \pm 43.31$ \\
Standard value & $<0.12$ & $0.07-0.11$ & $9-22$ & $80-87$ & $19-42$ \\
\hline
\end{tabular}

Notes: a total of 50 subjects with CS neck pain were treated with acupuncture. The needles were retained in the points for $60 \mathrm{~min}$ once every other day with 10 sessions constituting one course. The pulse diagram parameter was measured with pulse acquisition device based on image before and after the first acupuncture treatment and compared. Five patients (the difference value was 5 to 0 in the assessment scale) did not see an effect, 27 patients (the difference value was 13 to 6 in the assessment scale) had significant changes, and eighteen patients (the difference value was 23 to 14 in the assessment scale) had very significant changes.

\section{Discussion}

For CS, TCM considers that Qi and blood stasis and arthralgia are the predominant disease changes. Therefore, the clinical treatment for CS is focused on these two aspects. In China, the use of acupuncture in the treatment of CS neck pain has been suggested[14-17]. TCM pulse diagnosis is one of the major clinical diagnostic methods in TCM, but the descriptions of pulses are subject to the interpretation of individual doctors, and this lacks quantitative standardization. King et al [18] tried to quantify TCM pulse diagnosis but were unable to reflect pulse qualities adequately. We aimed to study objective PDP parameters before and after acupuncture treatment that reflect the blood-Qi changes of the body.

In our study, we found that the pulse wave in patients was disorganized and irregular before the first treatment, especially in the anterior half, which indicated the influence of the abnormal of Qi and blood caused by CS. However, the pulse wave became regular after treatment. Based on the physiological significance represented by the PDP changes, we found that the tone of the great vessels was lower, the arterial elasticity was improved, and the pulse normalized. This indicated that acupuncture can regulate vascular function, effectively relieve the compression of spinal nerves and vasculature. These changes can improve the dysfunction of Qi and blood caused by CS. Therefore, PADBI has important significance in the objective evaluation of acupuncture treatment for CS.

In addition, we found that the PDP was normalized after the tenth treatment in patients whose PDP was closer to normal after the first treatment. The PDP after the tenth treatment did not improve compared with that before the first treatment when the PDP had no obvious change from before to after the first acupuncture treatment. Therefore, acupuncture treatment was effective for patients if it was effective after the first treatment. The effect of acupuncture in the treatment of CS was positive if patients continued treatment. The acupuncture treatment was ineffective for patients if it was ineffective after the first treatment and it may not develop favorable therapeutic effects if patients continued treatment. After the tenth treatment, changes in PDP did not improve in five patients, which indicated that their CS symptoms were not improved.

Changes in pulse information can reflect changes in Yin-Yang, Qi-blood, the function of viscera, and it can reflect hemodynamic changes in the cardiovascular and circulatory system. The transition of traditional sphygmology from qualitative to quantitative was an important aspect of the objective and standardization of sphygmology. Standardization has important significance to the development of pulse diagnosis in TCM. According to analysis of PDP by time domain analysis, we found that the PDP significantly changed from before to after acupuncture treatment. The main manifestations of the time domain signals were disorganized in patients, but they became regular after treatment. This is because CS had relatively obvious influences on the central nervous system. Time domain analysis mainly analyzes the relationship between amplitude of pulse signals and phase of pulsation. Therefore, disorganized signals before the first treatment became regular after treatment. This change indicated that the compressive symptoms of the neck were improved and the central nervous system symptoms were relieved after acupuncture treatment[19]. 
In conclusion, changes in PDP parameters and symptoms in patients with CS after treatment were consistent, so PDP could be used to study standard treatment CS. Changes in PDP from before until after the first acupuncture treatment and the efficacy of late treatment were consistent. Therefore, PDP before and after an initial acupuncture treatment could be a predictive index of efficacy.

\section{References}

1. Rao RD, Currier BL, Albert TJ, et al. Degenerative cervical spondylosis: clinical syndromes, pathogenesis, and management. J Bone Joint Surg Am. 89(6) , 1360- 1378 (2007).

2. Butler JS, Oner FC, Poynton AR, et al. Degenerative cervical spondylosis: natural history, pathogenesis, and current management strategies. Adv Orthop.2012, 916987(2012).

3. Ferrara LA. The biomechanics of cervical spondylosis. Adv Orthop .2012, 493605(2012).

4. Binder A. Cervical spondylosis and neck pain. BMJ. 334(7592), 527-531(2007).

5. Trinh K, Cui X, Wang YJ. Chinese herbal medicine for chronic neck pain due to cervical degenerative disc disease. Spine. 35(24), 2121-2127(2010).

6. Avery RM. Massage therapy for cervical degenerative disc disease: alleviating a pain in the neck? Int J Ther Massage Bodywork . 5(3) ,41-46(2012).

7. Lauche R, Langhorst J, Dobos GJ, et al. Clinically meaningful differences in pain, disability and quality of life for chronic nonspecific neck pain - a reanalysis of 4 randomized controlled trials of cupping therapy. Complement Ther Med . 21(4),342-347(2013).

8. Bertozzi L, Gardenghi I, Turoni F, et al. Effect of therapeutic exercise on pain and disability in the management of chronic nonspecific neck pain: systematic review and meta-analysis of randomized trials. Phys Ther. 93(8), 1026-1036(2013).

9. $\mathrm{Fu} \mathrm{WB}$, Liang $\mathrm{ZH}$, Zhu XP, et al. Analysis on the effect of acupuncture in treating cervical spondylosis with different syndrome types. Chin J Integr Med. 15(6), 426-430(2009).

10. Liang ZH, Di Z, Jiang S, et al. The optimized acupuncture treatment for neck pain caused by cervical spondylosis: a study protocol of a multicentre randomized controlled trial. Trials.13, 107(2012).

11. Willich SN, Reinhold T, Selim D, et al. Cost effectiveness of acupuncture treatment in patients with chronic neck pain. Pain.125(1-2), 107-113(2006).

12. Sun Y, Chen Q. The 2nd symposium meeting on cervical spondylosis. China J Surg. 31(8), 472476(1993).

13. $\mathrm{Yu} \mathrm{W}$, Huo S. The paper introduces a Evaluation Method of lumbar function. Zhong Guo Kang Fu Yi Xue Za Zhi.13(5), 214-215(1998).

14. Guo YQ, Chen LY, Fu WB, et al. Clinically randomized controlled study on abdominal acupuncture for treatment of cervical spondylosis. Zhong Guo Zhen Jiu.27(9), 652-656(2007).

15. Liang $\mathrm{ZH}$, Yang $\mathrm{YH}, \mathrm{Yu} \mathrm{P}$, et al. Logistic regression analysis on therapeutic effect of acupuncture on neck pain caused by cervical spondylosis and factors influencing therapeutic effect. Zhong Guo Zhen Jiu.29(3), 173-176(2009).

16. Dong WK, Lin XH. Clinical observation on cervical spondylosis of neck type treated with acupuncture at original and terminal points of trapeziust. Zhong Guo Zhen Jiu.32(3),211214(2012).

17. Xu SJ, Liang ZH, Fu WB. Chronic neck pain of cervical spondylosis treated with acupuncture and moxibustion in terms of the heart and kidney theory: a randomized controlled trial. Zhong Guo Zhen Jiu .32(9) ,769-775(2012).

18. King EI, Cobbin D, Ryan D. The reliable measurement of radial pulse: gender differences in pulse profiles. Acupunct Med. 20(4), 160-167(2002).

19. Liu ZR, Li XX. On analysis of sphygmogram. Sci Sin B. 25(12), 1286-1295(1982). 